\title{
Direct inversion of the apparent complex-resistivity spectrum
}

\author{
J. Xiang $\ll$ N. B. Jones $\ll$ D. Cheng ${ }^{\ddagger}$, and F. S. Schlindwein $\ll$
}

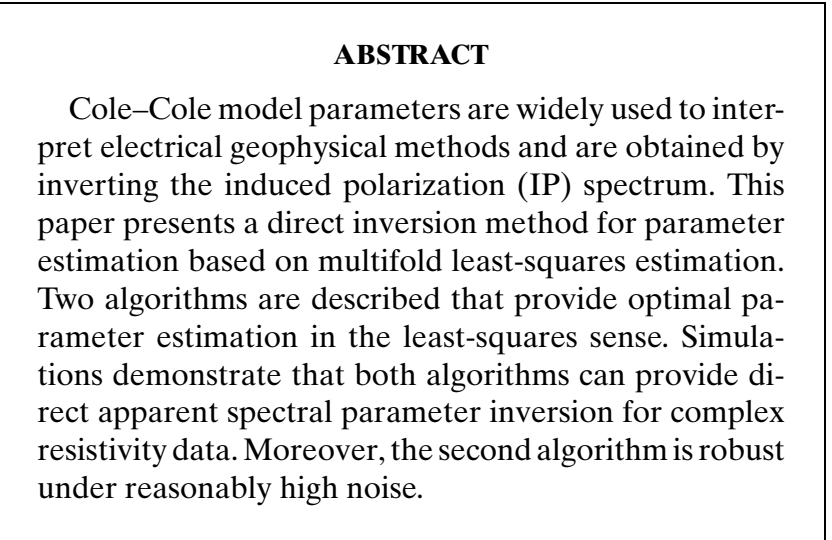

\section{INTRODUCTION}

Spectral induced polarization (IP) is widely used in geological surveys (He et al., 1995; He, 1996; Milson, 1996; Luo and Zhang, 1998). The interpretation of the IP data is often based on the Cole-Cole model (Cole and Cole, 1941; Luo and Zhang, 1998), under which the frequency behaviour of the complex IP impedance (or complex resistivity) is approximated by an equivalent network, as shown in Figure 1 (Major and Silic, 1981).

The impedance of the equivalent network is

$$
Z(\omega)=Z(0)\left(1 \ll m\left(1 \ll \frac{1}{1+(j \omega \phi)^{c}}\right)(\right.
$$

The complex resistivity expression of the Cole-Cole model (Luo and Zhang, 1998) is written

$$
\phi(\omega)=\phi_{0}\left(1 \ll m\left(1 \ll \frac{1}{1+(j \omega \phi)^{c}}\right),\right.
$$

where $\phi(\omega)$ is complex resistivity, $\phi_{0}$ is resistivity at zero frequency $(\omega=0), m$ is limited polarizability (or chargeability), $\phi$ is a time constant, $c$ is frequency dependence, and $\omega$ is angular frequency $(\mathrm{rad} / \mathrm{s})$. The chargeability $0 \ll m \ll 1$ and the requirement that the complex resistivity amplitude monotoni- cally decreases with increasing frequency restricts $c$ to the range $0 \ll c \ll 1$ (Major and Silic, 1981). Interpretation based on the Cole-Cole model requires the parameters $\phi_{0}, m, \phi$, and $c$ to be estimated (Luo and Zhang, 1998).

The existing methods of parameter estimation are generally based on nonlinear iterative inversion (Pelton et al., 1984, 1978; Jaggar and Fell, 1988; Luo and Zhang, 1998). There is a serious shortcoming to this approach: The convergence of an iterative inversion to a global minimum is not ensured because it depends on the initial guess for parameter values. Different starting values may yield different inversion results.

We propose a new direct inversion technique based on multifold least-squares estimation. The basic procedure is described by the following sequence. First, use substitution to eliminate the parameter $m$ from the equations. Second, use least-squares estimation to express the compound parameter $X=\phi^{c}$ as a function of $\phi_{0}$ and $c$, which is expressed as $\phi^{c}=X\left(\phi_{0}, c\right)$. Third, consider the reduced system of equations for $\phi_{0}$ and $c$, which is linear with respect to $\phi_{0}$. Then the secondfold least-squares estimation can be used to get a solution for $\phi_{0}$ as a function of $c$.

The final step is to substitute all the estimated parameters into the equations to get a set of linear equations of $X$. Both the coefficients and the unknowns, $X$, of the linear equations are functions of $c$. A square error can be constructed for the leastsquares solution of $X$ as a function of $c$. Since the parameter $c \in(0,1)$, the golden section technique is used to search for the minimum solution $c$. Finally, the least-squares technique is used to estimate $m$.

This inversion technique is based on a straightforward computation that identifies the four parameters simultaneously and guarantees a unique solution. Moreover, the solution is optimal in the least-squares sense, and the method can be applied easily to the case of multiple, linearly additive Cole-Cole models.

\section{DIRECT INVERSION OF THE COLE-COLE MODEL}

Consider inverting the Cole-Cole model [equation (2)]. That is, from experimental data estimate $\phi_{0}, m, \phi$, and $c$.

Assume $N+1$ data are obtained as $\left\{\left(\omega_{k}, \phi_{k}\right) \mid k=1,2, \ldots\right.$, $N+1\}$, where $\phi_{k}=\phi\left(j \omega_{k}\right), k=1,2, \ldots, N+1$. Rewrite equation (2) as a set of equations:

Manuscript received by the Editor February 24, 2000; revised manuscript received February 1, 2001.

University of Leicester, Department of Engineering, University Road, Leicester LE1 7RH,U.K.E-mail: jx5@le.ac.uk; nbj1@le.ac.uk; fss1@le.ac.uk. $\ddagger$ Institute of Systems Science, Chinese Academy of Sciences, Beijing 100080, P.R. China. E-mail: dcheng@iss03.iss.ac.cn.

« 2001 Society of Exploration Geophysicists. All rights reserved. 
$m\left(1 \ll \frac{1}{1+\left(j \omega_{k} \phi\right)^{c}}\left(=\frac{\phi_{0} \ll \phi_{k}}{\phi_{0}}, \quad k=1,2, \ldots, N+1\right.\right.$,

where $\phi_{k}$ is complex resistivity at $\omega=\omega_{k}$.

To reduce the number of parameters, we divide the $k$ th equation by the $(k+1)$ th equation for $k=1,2, \ldots, N$. After some algebra we get $N$ equations as

$\frac{\omega_{k}^{c} \ll \omega_{k+1}^{c}}{\omega_{k+1}^{c}+\left(j \omega_{k} \omega_{k+1} \phi\right)^{c}}=\frac{\phi_{k+1} \ll \phi_{k}}{\phi_{0} \ll \phi_{k+1}}, \quad k=1,2, \ldots, N$.

Taking the reciprocal, equation (4) is equivalent to

$\frac{\omega_{k+1}^{c}+\left(j \omega_{k} \omega_{k+1} \phi\right)^{c}}{\omega_{k}^{c} \ll \omega_{k+1}^{c}}=R_{k}+j I_{k}, \quad k=1,2, \ldots, N$,

where

$$
R_{k}+j I_{k}=\frac{\phi_{0} \ll \phi_{k+1}}{\phi_{k+1} \ll \phi_{k}}, \quad k=1,2, \ldots, N .
$$

To get the least-squares approximate solution of equation (7), we have to minimize the square error form $S$ :

$$
\min _{x} S=\sum_{k=1}^{N}\left(A_{k} X \ll B_{k}\right)^{2}+\sum_{k=1}^{N}\left(C_{k} X \ll I_{k}\right)^{2} .
$$

Setting $d S / d X=0$, the minimum point of the strictly positive $S$ is obtained as

$$
X=\frac{\sum_{k=1}^{N}\left(A_{k} B_{k}+I_{k} C_{k}\right)}{\sum_{k=1}^{N}\left(A_{k}^{2}+C_{k}^{2}(\right.} .
$$

Substituting the expressions for $A_{k}, B_{k}$, and $C_{k}$ into equation (11), after some simplification, yields

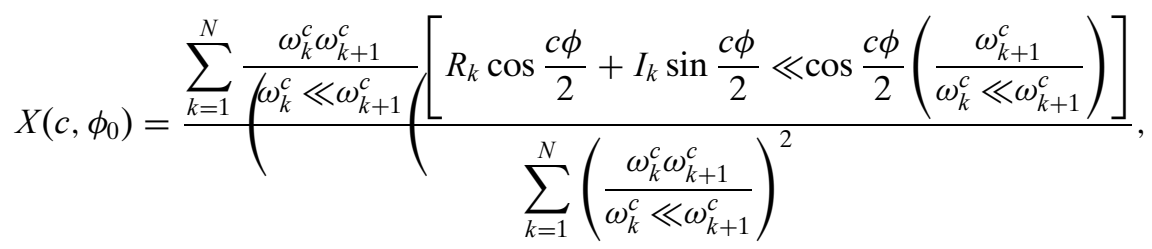

Note that data with repeated $\phi_{k}$ should be deleted to avoid ballooning to infinity.

Setting $\phi^{c}=X$ and separating the real and imaginary parts of equation (5) yields the following equations:

$$
\left(\begin{array}{l}
A_{k} X=B_{k} \\
C_{k} X=I_{k}
\end{array}, \quad k=1,2, \ldots, N\right.
$$

where

$$
\begin{aligned}
A_{k} & =\frac{\cos \frac{c \phi}{2} \omega_{k}^{c} \omega_{k+1}^{c}}{\omega_{k}^{c} \ll \omega_{k+1}^{c}}, \quad C_{k}=\frac{\sin \frac{c \phi}{2} \omega_{k}^{c} \omega_{k+1}^{c}}{\omega_{k}^{c} \ll \omega_{k+1}^{c}}, \\
D_{k} & =\frac{\omega_{k+1}^{c}}{\omega_{k}^{c} \ll \omega_{k+1}^{c}}
\end{aligned}
$$

and

$$
B_{k}=R_{k} \ll D_{k}
$$

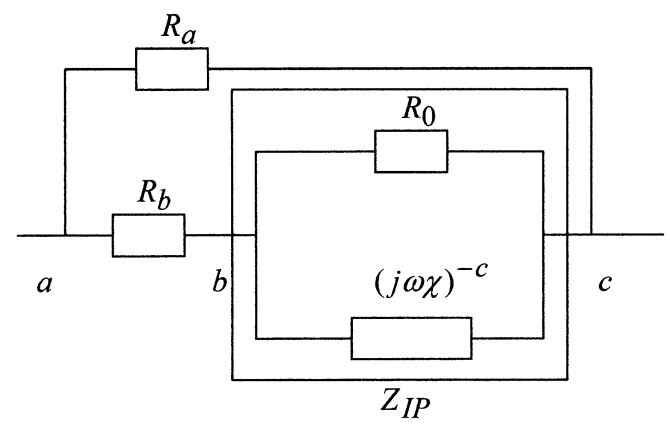

FIG. 1. Equivalent circuit of IP geophysical system where $X\left(c, \phi_{0}\right)$ emphasizes that the least-squares approximate solution for $\mathrm{X}$ depends on both $c$ and $\phi_{0}$.

Next, to estimate $\phi_{0}$ some new notation is convenient. Denote

$$
\phi_{0}=R_{0}+j I_{0}
$$

and

$$
\frac{1}{\phi_{k+1} \ll \phi_{k}}=\phi_{k}+j \phi_{k} ; \quad \frac{\phi_{k+1}}{\phi_{k+1} \ll \phi_{k}}=\phi_{k}+j \varphi_{k} .
$$

Then, from equation (6) the following expressions can be derived:

$$
R_{k}=\phi_{k} R_{0} \ll \phi_{k} I_{0} \ll \phi_{k}
$$

and

$$
I_{k}=\phi_{k} I_{0}+\phi_{k} R_{0} \ll \varphi_{k} .
$$

Substituting equations (15) and (16) into equation (12) provides

$$
X=P R_{0}+Q I_{0} \ll H,
$$

where

$$
P=\frac{\sum_{k=1}^{N} \frac{\omega_{k}^{c} \omega_{k+1}^{c}}{\omega_{k}^{c} \ll \omega_{k+1}^{c}}\left(\cos \frac{c \phi}{2} \phi_{k}+\sin \frac{c \phi}{2} \phi_{k}\right.}{\sum_{k=1}^{N}\left(\frac{\omega_{k}^{c} \omega_{k+1}^{c}}{\omega_{k}^{c} \ll \omega_{k+1}^{c}}\right)^{2}},
$$




$$
Q=\frac{\sum_{k=1}^{N} \frac{\omega_{k}^{c} \omega_{k+1}^{c}}{\omega_{k}^{c} \ll \omega_{k+1}^{c}}\left(\sin \frac{c \phi}{2} \phi_{k} \ll \cos \frac{c \phi}{2} \phi_{k}\right.}{\sum_{k=1}^{N}\left(\frac{\omega_{k}^{c} \omega_{k+1}^{c}}{\omega_{k}^{c} \ll \omega_{k+1}^{c}}\right)^{2}},
$$

and

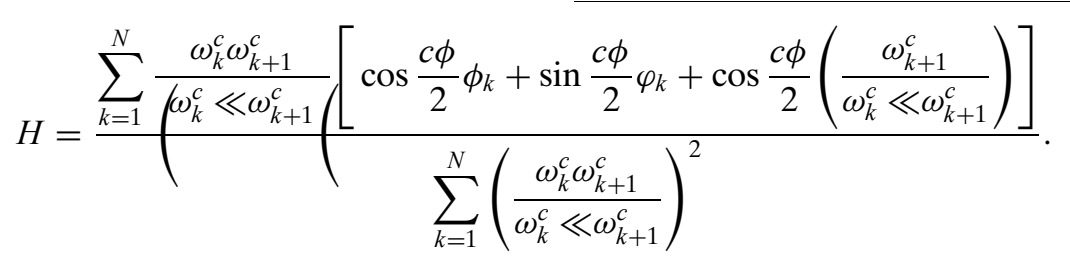

To determine $c$, we minimize $S(c)$. That is,

$$
\min _{c}\{S(c) \mid 0<c<1\} .
$$

Since $c \in(0,1)$, it lies on a restricted range. So we use the golden section method to search for the optimal solution (Press et al., 1986).

After the optimal $c$ (denoted $c^{4}$ ) is obtained, equation (25) [or equation (28) in the real case] provides the best estimate of
Applying equations (17)-(20) to equation (7) produces

$$
\left(\begin{array}{l}
A_{k}\left(P R_{0}+Q I_{0} \ll H\right)=\phi_{k} R_{0} \ll \phi_{k} I_{0} \ll \phi_{k} \ll D_{k} \\
C_{k}\left(P R_{0}+Q I_{0} \ll H\right)=\phi_{k} I_{0}+\phi_{k} R_{0} \ll \varphi_{k}
\end{array} .\right.
$$

Equation (21) can be expressed in matrix form as

$$
M(c)\left(\begin{array}{c}
R_{0} \\
I_{0}
\end{array}(=L(c),\right.
$$

where

$$
M(c)=\left(\begin{array}{ll}
A_{k} P \ll \phi_{k} & A_{k} Q+\phi_{k} \\
C_{k} P \ll \phi_{k} & C_{k} Q \ll \phi_{k}
\end{array}\right) \quad k=1, \ldots, N
$$

and

$$
L(c)=\left(\begin{array}{c}
A_{k} H \ll \phi_{k} \ll D_{k} \\
C_{k} H \ll \varphi_{k}
\end{array}\right) \quad k=1, \ldots, N .
$$

Here we use $M(c)$ and $L(c)$ to emphasize they depend on $c$ only. Now the least-squares solution of equation (22) is

$$
\left(\begin{array} { c } 
{ R _ { 0 } } \\
{ I _ { 0 } }
\end{array} \left(=\left[M^{T}(c) M(c)\right]^{\ll 1} M^{T}(c) L(c) .\right.\right.
$$

Using equation (25), the square error of equation (7) becomes

$$
\begin{aligned}
S(c)= & \left(M(c)\left[M^{T}(c) M(c)\right]^{\ll 1} M^{T}(c) L(c) \ll L(c)\right)^{T} \\
& \ll\left(M(c)\left[M^{T}(c) M(c)\right]^{\ll 1} M^{T}(c) L(c) \ll L(c)\right) .
\end{aligned}
$$

In practice, we can assume that $\phi_{0}$ is a real number. That is, $\phi_{0}=R_{0}$ and $I_{0}=0$. Then equation (23) can be replaced by

$$
M(c)=\left(\begin{array}{c}
A_{k} P \ll \phi_{k} \\
C_{k} P \ll \phi_{k}
\end{array}\right), \quad k=1, \ldots, N
$$

and

$$
\phi_{0}=R_{0}=\left[M^{T}(c) M(c)\right]^{\ll 1} M^{T}(c) L(c) .
$$

$\phi_{0} ; X=\phi^{c}$ can be obtained by equation (11). The estimation of $\phi$ follows easily.

Finally, equation (3) can be used to estimate $m$. Since $m$ is real and $m>0$, equation (3) can be rewritten as

$m \ll \frac{1}{1+\left(j \omega_{k} \phi\right)^{c}} k=\left(\frac{\phi_{0} \ll \phi_{k}}{\phi_{0}} k \quad k=1,2, \ldots, N+1\right.$.

Now, for this set of real equations the technique used in equations (7)-(11) can be used again for obtaining a least-squares solution as

$$
m=\frac{\sum_{k=1}^{N+1}\left(\frac{\left(j \omega_{k} \phi\right)^{c}}{1+\left(j \omega_{k} \phi\right)^{c}}\right)\left(\frac{\phi_{0} \ll \phi_{k}}{\phi_{0}}\right)}{\left(\sum_{k=1}^{N+1} \frac{\left(j \omega_{k}\right.}{\left(1+\left(j \omega_{k} \phi\right)^{c}\right.}\right)}
$$

\section{ALGORITHMS AND NUMERICAL EXAMPLES}

\section{Algorithm 1}

Summarizing the discussion in the last section, an algorithm can be obtained as follows. First, use equation (14) to get $\phi_{k}, \phi_{k}, \phi_{k}$, and $\varphi_{k}, k=1, \ldots, N$. Second, apply the golden section algorithm (or any alternative minimization method to find optimal $c$ ) to choose $c_{1}, c_{2}$. Third, use equations (8)-(10) to get $A_{k}\left(c_{1}\right), A_{k}\left(c_{2}\right), C_{k}\left(c_{1}\right), C_{k}\left(c_{2}\right), D_{k}\left(c_{1}\right)$, and $\quad D_{k}\left(c_{2}\right), k=1, \ldots$, $N+1$, respectively. Fourth, use equations (18)-(20) to get $P\left(c_{1}\right), P\left(c_{2}\right), Q\left(c_{1}\right), Q\left(c_{2}\right), H\left(c_{1}\right)$, and $H\left(c_{2}\right)$, respectively. Fi$\mathrm{fth}$, use equations (22) [or (24)] and (23) to construct $M\left(c_{1}\right), M\left(c_{2}\right)$ and $L\left(c_{1}\right), L\left(c_{2}\right)$, respectively.

The sixth and final step in this algorithm is to use equation (26) to calculate $S\left(c_{1}\right)$ and $S\left(c_{2}\right)$ and to compare them to decide if a convergence criterion for the solution has been satisfied. If $\left|c_{1} \ll c_{2}\right|<\varepsilon$ for a given $0<\varepsilon \ll 1$, stop. Otherwise, use the golden section algorithm to choose a new point and set new $c_{1}, c_{2}$. Then go back to step 3 .

We have written a Matlab program that performs this algorithm. The following example illustrates the accuracy of the approximation. 
Example 1.-Use a set of given parameters as in Luo and Zhang (1998):

$$
c=0.25, \quad \phi_{0}=25, \quad \phi=100, \quad m=0.5 .
$$

Take the sampling frequencies as

$$
\omega_{k}=2^{k \ll 13}, \quad k=1,2, \ldots, 20,
$$

that is, from $\omega=2^{\ll 12}$ to $\omega=2^{+7} \mathrm{rad} / \mathrm{s}$. Use equation (2) to get synthetic data values of $\phi_{k}=\phi\left(j \omega_{k}\right)$. Then invert the data set. The results using the above algorithm are shown in Tables 1 and 2 (where $c$ is the estimated value and $E_{c} \%$ is the relative error, etc.).

McInnes (personal communication, 2000) points out that the above algorithm is sensitive to noise in the observed apparent complex resistivity data. Motivated by this observation, we modified the performance criterion $S$ to optimize $c$. Let $S$ be the real square error of the estimation

$$
S(c)=\sum_{k=1}^{N+1}\left|\phi\left(\omega_{k}\right) \ll \phi_{c}\left(\omega_{k}\right)\right|^{2} .
$$

\section{Algorithm 2}

We modified algorithm 1 as follows. Using $c, \phi_{0}(c)$, and $\phi(c)$, we estimated $m(c)$ first. Then we used the estimated parameters and Cole-Cole model to calculate the model response $\phi_{c}\left(\omega_{k}\right)$. Equation (32) can be used to calculate the model response misfit. Finally, a single-variable minimization algorithm can be used to find the best estimation of $c$.

To perform this refined new algorithm, you repeat the first five steps for algorithm 1 . As the sixth step, use equations (28), (17), and (31) to find $\phi_{0}, \phi$, and $m$, respectively. For the supposed $c$, the corresponding model is built. As the seventh and final step, use equation (32) to calculate $S\left(c_{1}\right)$ and $S\left(c_{2}\right)$. Compare them to decide if a convergence criterion for the solution has been satisfied. That is, if $\left|c_{1} \ll c_{2}\right|<\varepsilon$ for a given $0<\varepsilon \ll 1$, stop. Otherwise use the golden section algorithm to choose a new point and set new $c_{1}, c_{2}$. Then go back to step 3 .

It is likely that the direct Cole-Cole inversion function $S(c)$ will have multiple local minima with some data sets. Then the interval $(0,1]$ can be divided into several subintervals, and the algorithm can be used over each subinterval to find the real minimum.

The algorithm is described by Figure 2, which consists of two essential parts. The first is the multifold, least-squares estimation, which uses the assumed parameter $c$ and the field data to estimate $\phi_{0}(c), m(c)$, and $\phi(c)$. Part 2 is the golden section algorithm.

To represent the accuracy of the approximation, we use a square error criterion. That is, let $p(n)$ and $q(n), n=1$, $2, \ldots, N$, be the field data and the theoretical data obtained from the Cole-Cole model, respectively. Then the square error is defined as $E_{s}=\sum_{n=1}^{N}|p(n) \ll q(n)|^{2}$, which is the same error criterion minimized by Jaggar and Fell (1988) to estimate their model parameters.

Using algorithm 2, we revisit example 1. When there is no measurement noise, the parameters can be recovered precisely without visible error (within Matlab's precision for long variables, that is, $\varepsilon<0.000015)$. When white noise is added to the complex resistivities, the values of estimated parameters will change. The algorithm is said to be robust if this sensitivity of the parameters to measurement noise is small. Table 2 illustrates the robustness of the algorithm.

A relative noise level of $10 \ll 2 \%$ represents a $0.1 \mathrm{mrad}$ IP phase error for the first harmonics, which is good-quality data for typical dipole-dipole surveys (S. McInnes, personal communication, 2000). From Table 2 one can see that the robustness of algorithm 2 is adequate for practical use.

The range of frequencies used affects the accuracy of the approximation. In the example above, if we narrow the range of frequencies to $N=12$ and $\omega=2^{k}, k=\ll 6, \ll 5, \ldots, 5$, the range of the frequency, in hertz, is $2.49 \ll 10^{\ll 3}<f<5$.1. Then the related estimation is obtained as in Table 3, which shows the accuracy is still acceptable.

\section{FIELD DATA INYERSION}

Jaggar and Fell (1988) report $N=19$ data values, but highfrequency data values have large uncertainty. Table 4 shows the results estimated via algorithm 2 by using the first $N=12$,

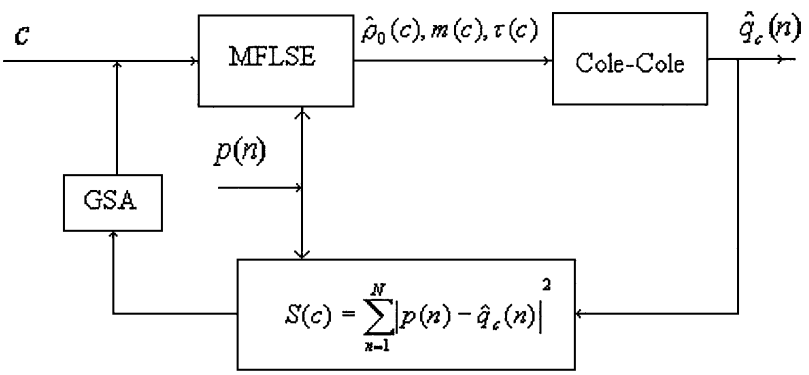

FIG. 2. Flow chart of algorithm 2 .

Table 1. Estimation for parameters with different accuracy.

\begin{tabular}{llllccccc}
\hline$\varepsilon$ & $\mathrm{C}$ & $E_{c}(\%)$ & $\phi_{0}$ & $E_{\phi_{0}}(\%)$ & $\phi$ & $E_{\phi}(\%)$ & $m$ & $E_{m}(\%)$ \\
\hline 0.01 & 0.2528 & 1.327 & 24.887 & 0.491 & 92.391 & 7.61 & 0.4971 & 0.589 \\
0.001 & 0.2498 & $<0.0001$ & 25.009 & 0.03 & 100.572 & 0.572 & 0.5002 & 0.042 \\
0.0004 & 0.25 & $<0.0001$ & 25 & $<0.0001$ & 100 & $<0.0001$ & 0.5 & $<0.0001$ \\
\hline
\end{tabular}

Table 2. Estimation for parameters with random measurement error.

\begin{tabular}{ccccc}
\hline $\begin{array}{c}\text { Measure noise } \\
\text { level }(\%)\end{array}$ & Estimated c & Estimated $\phi_{0}$ & Estimated $\phi$ & Estimated $m$ \\
\hline $10^{\ll}$ & 0.2502 & 24.9988 & 100.2814 & 0.4998 \\
$10^{\ll}$ & 0.2508 & 24.9732 & 99.0512 & 0.499 \\
1 & 0.2764 & 23.8827 & 75.7240 & 0.4572 \\
\hline
\end{tabular}


$13, \ldots, 17$ data values. The best-fit model suggested by Jaggar and Fell (1988), data size 19 on Table 4, is compared with the models found with our methods in Table 5 .

Another advantage of our algorithm is that it does not need an initial guess. Table 6 shows that the ridge regression method adopted by Jaggar and Fell (1988) clearly depends on the initial guess. It might only find a local minimum. Table 6 shows three models $(N-13, N-16, N-17)$ provide better estimation than the J-F model, even over all 19 sampling data points.

If we ignore the last two outlier data values, then the model errors over the set of $N=12-17$ reliable data values are shown in Table 7. So from both Tables 6 and 7 we can see that model $\mathrm{N}-16$ seems to best fit the field data. We therefore can choose $N-16$ as our finally accepted model. Figures 3 and 4 compare the field data with the theoretical data.

\section{CONCLUSION}

A direct inversion technique for the Cole-Cole model was presented. The method is based on a multifold least-squares estimation combined with an optimal searching technique. Two step-by-step algorithms were derived and described via several numerical examples. The advantages of the final new approach are

1) the estimation is optimal in the least-squares sense,

2) the existence of a solution is guaranteed,

3) the algorithm is straightforward and simple,

4) no iterative operator intervention is required, and

5) amplitude and phase can be fit simultaneously.

Using the field data provided by Jaggar and Fell (1988), our algorithm has been verified and a detailed comparison with their existing algorithm has been completed. The results show the advantages of the new algorithm.

\section{ACKNOWLEDGMENTS}

The authors thank the Royal Society of U.K. for funding J. X. and Royal Society Fellowship G1998020308 for D.C. Special thanks are owed to Peter Maguire and M.A. Meju for their kind cooperation to the project associated with the fund. The authors acknowledge J. A. Twiddle on the use of the Matlab/Simulink software and S. McInnes for excellent com- ments that showed us the lack of robustness in the previous version of the algorithm. That challenged us to improve the technique presented here.

\section{REFERENCES}

Cole, K. S., and Cole, R. H., 1941, Dispersion and absorption in dielectrics, I: Alternating current field: J. Chem. Phys., 1, 341-351.

He, J., 1996, Frequency domain electrical methods for searching resources of non-ferrous metals in China: Internat. J. Soc. Mat. Eng. Res., 4, No. 1, 72

He, J., Li, D., and Tang, J., 1995, Equivalent circuit of non-linear effect of spectral IP: Trans. Nonferrous Metals Soc. China, 5, 1-7.

Table 5. Comparison of the model error within sampling data.

\begin{tabular}{ccc}
\hline Data size $N$ & Jagger-Fell Model error & Our model error \\
\hline 12 & 0.0876 & 0.0029 \\
13 & 0.0998 & 0.0036 \\
14 & 0.1210 & 0.0068 \\
15 & 0.1506 & 0.0076 \\
16 & 0.1729 & 0.0156 \\
17 & 0.1794 & 0.0565 \\
\hline
\end{tabular}

Table 6. Comparison of the model errors for all data values.

\begin{tabular}{cc}
\hline Model & Overall error \\
\hline Jaggar-Fell & 0.3164 \\
N-12 & 0.3250 \\
N-13 & 0.1684 \\
N-14 & 0.3732 \\
N-15 & 0.3595 \\
N-16 & 0.1682 \\
N-17 & 0.1930 \\
\hline
\end{tabular}

Table 7. Comparison of the model error over reliable data.

\begin{tabular}{cc}
\hline Model & Overall error \\
\hline Jaggar-Fell & 0.1794 \\
N-12 & 0.0378 \\
N-13 & 0.0387 \\
N-14 & 0.0460 \\
N-15 & 0.0416 \\
N-16 & 0.0191 \\
N-17 & 0.0567 \\
\hline
\end{tabular}

Table 3. Parameter estimation with a narrower frequency range.

\begin{tabular}{cccc}
\hline $\begin{array}{c}\text { Measure noise } \\
\text { level }(\%)\end{array}$ & Estimated c & Estimated $\phi_{0}$ & Estimated $\phi$ \\
\hline 0 & 0.25 & 25 & 100 \\
$10^{\ll 2}$ & 0.2491 & 25.053 & 102.6495 \\
$10^{\ll 1}$ & 0.2472 & 25.1652 & 108.8144 \\
1 & 0.2374 & 23.6537 & 48.3621 \\
\hline
\end{tabular}

Table 4. Estimated parameters via field data.

\begin{tabular}{ccccc}
\hline Data size $N$ & Estimated $c$ & Estimated $\phi_{0}$ & Estimated $\phi$ & Estimated $m$ \\
\hline 12 & 0.6896 & 21.9901 & 0.0013 & 0.1235 \\
13 & 0.6702 & 21.994 & 0.0010 & 0.1343 \\
14 & 0.6812 & 21.992 & 0.0013 & 0.1223 \\
15 & 0.6824 & 21.9902 & 0.0012 & 0.1226 \\
16 & 0.6502 & 21.9907 & 0.0009 & 0.1341 \\
17 & 0.6502 & 21.9907 & 0.0008 & 0.1373 \\
19 & 0.691 & 21.90 & 0.001165 & 0.128 \\
\hline
\end{tabular}




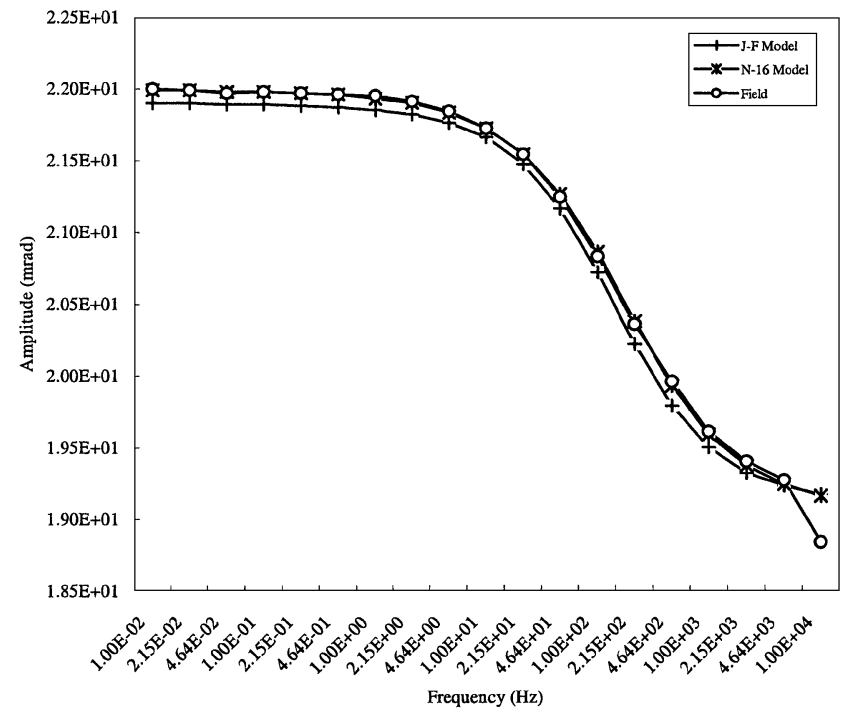

FIG. 3. Comparison of amplitude.

Jaggar, S. R., and Fell, P. A., 1988, Forward and inverse Cole-Cole modeling in the analysis of frequency domain electrical impedance data: Expl. Geophys., 19, 463-470.

Luo, Y., and Zhang, G., 1998, Theory and application of spectral induced polarization: Soc. Expl. Geophys.

Major, J., and Silic, J., 1981, Restrictions on the use of Cole-Cole dispersion models in complex resistivity interpretation: Geophysics, 46,

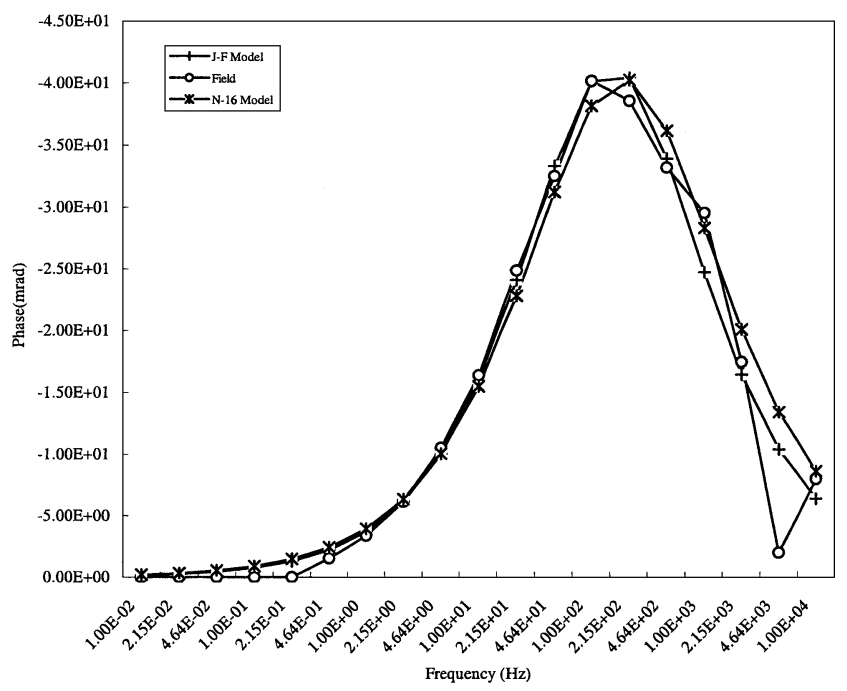

FIG. 4. Comparison of phasez

Milson, J., 1996, Field geophysics: John Wiley \& Sons, Inc.

Pelton, W. H., Smith, B. D., and Sill, W. R., 1984, Interpretation of complex resistivity and dielectric data, part II: Geophys. Trans., 29, No. 4, 11-45.

Pelton, W. H., Ward, P. G., Sill, W. R., and Nelson, P. H., 1978, Mineral discrimination and removal of inductive coupling with multifrequency IP: Geophysics, 43, 588-609.

Press, W. H., Flannery, B. P., Teukolsky, S. A., and Vetterling, W. T. 1986, Numerical recipes in C: Cambridge Univ. Press. 